\title{
Minimal residual disease by either flow cytometry or cytogenetics prior to an allogeneic hematopoietic stem cell transplant is associated with poor outcome in acute myeloid leukemia
}

Maxim Norkin', Lakshmikanth Katragadda', Fei Zou², Sican Xiong ${ }^{2}$, Myron Chang ${ }^{2}$, Yunfeng Dai ${ }^{2}$, Jack W. Hsu', Jan S. Moreb ${ }^{1}$, Helen Leather ${ }^{1}$, Hemant S. Murthy ${ }^{1}$, Nosha Farhadfar', Ying Li ${ }^{3}$, Robert Hromas (1)', Randy A. Brown ${ }^{1}$, Christopher R. Cogle ${ }^{1}$ and John R. Wingard ${ }^{1}$

\begin{abstract}
Relapsed acute myeloid leukemia (AML) is a significant challenge after allogeneic hematopoietic cell transplant (HCT). Multiparameter flow cytometry (MFC), conventional cytogenetics (CG), and fluorescence in situ hybridization (FISH) are routinely performed on bone marrow specimens prior to HCT to assess disease status. We questioned the extent by which pre-HCT evidence of minimal residual disease (MRD) detected by these standard assays, corresponded with post-HCT relapse. We conducted a single center, retrospective study of $166 \mathrm{AML}$ patients who underwent HCT. Thirtyeight of one hundred sixty-six (23\%) patients in complete remission (CR) or CR with incomplete count recovery (CRi) had MRD detectable by MFC, CG, or FISH. MRD was more frequently seen in patients with poor risk karyotype at diagnosis $(P=0.011)$. MRD-negative patients $\left(M^{2} D^{\text {neg }}\right)$ had significantly longer overall survival $(O S)$ and relapse-free survival than patients who were MRD positive $\left(M_{R} D^{\text {pos }}\right)\left(P=0.002\right.$ and 0.013 , respectively). In patients with MRD ${ }^{\text {pos }}$ prior to $\mathrm{HCT}$, the presence of acute graft vs. host disease (GVHD) (grade $\geq 2$ ) or chronic GVHD significantly improved progression free survival (PFS) (hazard ratio $(H R)=0.053$ (95\% confidence interval $(C l): 0.01-0.279), P=0.0005$ ) and OS $(\mathrm{HR}=0.211$ (95\% Cl: 0.081-0.547), $P=0.0014)$.
\end{abstract}

\section{Introduction}

There have been only modest improvement in outcomes in acute myeloid leukemia (AML) over the last several decades. While most of this progress has come due to advances in supportive care, some benefit has resulted from better prognostication of AML and risk-adapted therapy $^{1,2}$. Approximately $60-80 \%$ of AML patients

\footnotetext{
Correspondence: Maxim Norkin (norkinm@ufl.edu)

'Division of Hematology and Oncology, Department of Internal Medicine,

College of Medicine, University of Florida, Gainesville, FL, USA

${ }^{2}$ Department of Biostatistics, College of Medicine, University of Florida,

Gainesville, FL, USA

Full list of author information is available at the end of the article
}

achieve $\mathrm{CR}$ after induction chemotherapy, although the majority eventually relapse due to surviving myeloblasts that are not detectable by light microscopic examination ${ }^{3}$. Using supplemental techniques such multiparameter flow cytometry (MFC), conventional cytogenetics (CG) and fluorescence in situ hybridization (FISH) we are able to detect the presence of low-level disease, commonly termed minimal residual disease (MRD). There is considerable interest in the impact of MRD status at the time of hematopoietic cell transplant (HCT) to predict postHCT outcomes.

Several studies have reported adverse outcomes in patients who are $\mathrm{MRD}^{\text {pos }}$ prior to allogeneic $\mathrm{HCT}^{4-6}$. In 
these studies 10-color MFC was exclusively used for identifying MRD status. The significance of persistent abnormal karyotype as evidence of MRD is not as clear, with a limited number of studies with small sample size that produced contradictory results ${ }^{7-13}$. In addition, it remains unclear if intensification of the conditioning regimen and development graft-vs.-leukemia (GVL) effect accompanying graft vs. host disease (GVHD) are capable of mitigating the adverse impact of MRD on post-HCT outcomes.

In this study, we retrospectively evaluated the effect of pre-HCT MRD ${ }^{\text {pos }}$, by either 4-color MFC and/or CG/ FISH on outcomes of AML patients undergoing HCT at our institution.

\section{Patients and methods}

The study was approved by University of Florida institutional review board (UF IRB201400410). We retrospectively reviewed all AML patients aged $\geq 18$ years in CR or CRi prior to HCT between January 2001 and January 2014. Surviving patients had at least 1 year of follow-up. AML disease status was assessed prior to HCT for all the patients by morphologic examination, 4-color MFC, CG, and FISH testing in bone marrow aspirate and biopsy specimens. CR was defined as bone marrow myeloblasts $<5 \%$ by morphology in a normocellular bone marrow, absence of extra medullary leukemia, neutrophil count $>1000 / \mu \mathrm{L}$, and a platelet count $>100,000 / \mu \mathrm{L}^{14}$. CRi was defined as meeting all the criteria for $\mathrm{CR}$ except for incomplete peripheral blood count recovery (neutrophils and/or platelets). Additional patient information, including time to relapse and mortality, were obtained from medical records, or from institutional database containing information regarding disease status, complications and survival through annual phone interviews.

Patient-specific, disease-specific, and HCT-specific variables known to impact AML prognosis were collected including ${ }^{15}$ : age ( $<40$ vs. $41-60$ vs. $>60$ years); secondary AML diagnosis, defined as AML that developed after a history of antecedent hematologic disorder or after prior treatment with systemic chemotherapy and/or radiotherapy for a previous unrelated cancer; cytogenetic risk category (good, intermediate, poor) defined as per NCCN guidelines ${ }^{16}$; remission status at the time of HCT (first remission (CR1) vs. >CR1); achievement of CR vs. CRi; duration of CR1 ( $\leq 12$ months vs. $>12$ months); intensity of HCT conditioning regimen ${ }^{17}$ (myeloablative vs. reduced intensity); donor type (matched sibling vs. others); and donor to recipient gender match (female donor-male recipient vs. other).

Assessment of MRD was performed on bone marrow aspirate samples obtained within 28 days prior to HCT using at least one of the following modalities: 4-color MFC, karyotyping utilizing CG, and FISH. Patients with evidence of disease by either technique were classified as being $M R D^{\text {pos }}$, and patients who had absence of disease using these techniques, categorized as $M R D^{\text {neg }}$.

MFC was performed on bone marrow specimens using monoclonal antibodies that were methodically used either as a large panel if the patient was newly evaluated or as a limited but targeted panel based on previously known patient-specific leukemia immune phenotype. MRD was reported as a percentage of CD45 positive white blood cells (WBCs) and was labeled MRD ${ }^{\text {pos }}$ if leukemic cells account for $\geq 0.1 \%$ of the analyzed total WBCs.

CG was performed using standard G-banding methods on 20 metaphase cells. FISH was reported as a percentage of abnormal nuclei among the examined 300 interphase nuclei. MRD ${ }^{\text {pos }}$ by CG was defined as abnormal karyotype seen in at least two metaphase cells, or less than two cells if it was a previously known abnormality for the given patient. FISH positivity of a prior known abnormality was labeled $\mathrm{MRD}^{\mathrm{pos}}$. For our analysis, we combined the list of patients with $\mathrm{MRD}^{\mathrm{pos}}$ by either CG or FISH.

As polymerase chain reaction (PCR) results for nucleophosmin (NPM1), fms-related tyrosine kinase 3 (FLT3), and CCAAT/enhancer binding protein alpha (CEBPA) mutations were not available for all patients, they were not used for assessment of MRD.

\section{Statistical analyses}

Patient, disease, and transplant characteristics of $M R D^{\text {pos }}$ and $M R D^{\text {neg }}$ groups were compared by chisquare test and Wilcoxon rank-sum test, as appropriate. Relapse-free survival (RFS) was defined as time to relapse from HCT. Patients who are alive and continue to be in remission were censored for RFS at last day of contact. Patients who died without relapse were also censored for RFS at the day of death. We performed multivariate risk factor analysis by proportional sub-distribution hazards regression model. In risk factor analysis, RFS was used as the response variable. Patients who were not in $\mathrm{CR}$ or $\mathrm{CRi}$ at HCT were excluded for the analysis. Complete remission (CR vs. CRi) before $\mathrm{HCT}$, karyotype risk category at diagnosis, duration of first remission $(\leq 12$ months vs. $>12$ months), CR status (CR1 vs. >CR1), conditioning regimen (myeloablative vs. others), age at $\mathrm{HCT}$, gender, type of AML (de novo vs. secondary), donor type (matched sibling vs. other), and donor:recipient sex match (female donor:male recipient vs. all other gender combinations) were used as explanatory variables. A backward selection procedure at the 0.20 significance level was applied to form the final model including only important explanatory variables. Due to the nature of competing risk between relapse and death in HCT, we also estimated the distribution of RFS by the method of cumulative incidence rate. We compared distributions of RFS between patients with $M_{R} D^{\text {pos }}$ and MRD neg by Gray test ${ }^{18,19}$. 
Similarly, within $\mathrm{MRD}^{\mathrm{pos}}$ patients, we compared distributions of RFS between those with and without acute and chronic GVHD using Gray test.

Overall survival (OS) was defined as time from HCT to death. Surviving patients were censored at date of last contact for OS. The Kaplan-Meier method was applied to estimate OS distribution. OS distributions between patients with $M R D^{\text {pos }}$ and $M R D^{\text {neg }}$ were compared by logrank test. The multivariate Cox proportional hazards model and backward selection procedure were applied for the risk factor analysis with OS as the response variable and with the same explanatory variables as in the analysis for RFS. Within the cohort of MRD pos patients we also compared distributions of OS between patients with and without acute and chronic GVHD using the Kaplan-Meier method. Data analyses were performed using R software ${ }^{20}$ ) and SAS software version 9.4 (SAS Institute Inc., Cary, NC, USA).

\section{Results}

A total of 166 consecutive AML patients were identified as having CR/CRi prior to HCT and were included in the study. Baseline characteristics of patients are shown in Supplementary Table 1. The median follow-up among patients who were 46 months (range, 13-103).

Thirty-eight (23\%) patients were MRD ${ }^{\text {pos }}$ by either MFC or CG/FISH. MRD was positive by MFC in 21 of 38 (55\%) patients, by CG or FISH in 25 of 38 (66\%) patients, and by both in 8 of $38(21 \%)$ patients. Disease and HCT characteristics of the MRD ${ }^{\text {pos }}$ and $M R D^{\text {neg }}$ groups are shown in Table 1. A significantly higher percentage of $M R D^{\text {pos }}$ patients had a poor risk karyotype at the time of AML diagnosis ( $47 \%$ vs. $26 \% ; P=0.011)$, and had a trend toward having CRi at the time of HCT (26\% vs. $14 \%$; $P=$ 0.077 ) in comparison to $M D^{\text {neg }}$ patients. The remaining variables known to be prognostic in AML were evenly balanced between the two groups.

\section{OS and RFS}

OS curves for patients with $M R D^{\text {pos }}$ and MRD ${ }^{\text {neg }}$ are presented in Fig. 1. Patients who were $M R D^{\text {neg }}$ had significantly longer $\mathrm{OS}$ than patients who were $\mathrm{MRD}^{\text {pos }}$ (997 days (95\% CI: $477-3096)$ vs. 240 days (95\% CI: 172-402); $P=0.002$ ). We performed a multivariate analysis using Cox proportional hazards model and backward selection procedure with $\mathrm{OS}$ as a response variable. The results revealed that patients who were $\mathrm{MRD}^{\text {neg }}(\mathrm{HR}=$ 0.55 (95\% CI: $0.35-0.87) ; P=0.011$ ), good or intermediate risk karyotype (HR $=0.51$ (95\% CI: 0.33-0.79), $P=0.003$ ) and $\mathrm{CR}$ as compared to $\mathrm{CRi}(\mathrm{HR}=0.52$ (95\% CI: $0.31-0.87), \quad P=0.013)$ had significantly better OS (Table 2).

The cumulative incidence of relapse for patients with $M_{R D}{ }^{\text {pos }}$ and $M R D^{\text {neg }}$ are shown in Fig. 2. Patients who
Table 1 Comparison of pre-HCT variables between $M^{\text {MRos }}{ }^{\text {pos }}$ and ${ }^{\text {neg }}$ cohorts

\begin{tabular}{|c|c|c|c|c|}
\hline Covariate & Label & $\begin{array}{l}\text { MRD + } \\
\mathrm{N}(\%)\end{array}$ & $\begin{array}{l}\text { MRD - } \\
\text { N (\%) }\end{array}$ & P-value \\
\hline Total number $(M)$ & & $38(23)$ & $128(77)$ & \\
\hline \multirow[t]{3}{*}{ Age } & $<40$ & $8(21)$ & $20(16)$ & \multirow{3}{*}{0.708} \\
\hline & $40-59$ & $20(53)$ & $69(54)$ & \\
\hline & $\geq 60$ & $10(26)$ & $39(30)$ & \\
\hline \multirow[t]{2}{*}{ Gender } & $\mathrm{F}$ & $20(53)$ & $60(47)$ & \multirow{2}{*}{0.560} \\
\hline & M & $18(47)$ & $67(53)$ & \\
\hline \multirow[t]{3}{*}{ Cytogenetics } & Favorable/ & $20(53)$ & $95(74)$ & \multirow{3}{*}{0.011} \\
\hline & intermediate risk & & & \\
\hline & Poor risk & $18(47)$ & $33(26)$ & \\
\hline \multirow{2}{*}{$\begin{array}{l}\text { Transplant done in } \\
\text { first remission (CR1) }\end{array}$} & CR1 & $28(74)$ & $97(76)$ & \multirow{2}{*}{0.792} \\
\hline & $>C R 1$ & $10(26)$ & $31(24)$ & \\
\hline \multirow{2}{*}{$\begin{array}{l}\text { Transplant for } \\
\text { relapsed } A M L \text { : } \\
\text { duration of } C R 1\end{array}$} & $>12$ months & $31(82)$ & $113(88)$ & \multirow{2}{*}{0.285} \\
\hline & $\leq 12$ months & $7(18)$ & $15(12)$ & \\
\hline \multirow[t]{2}{*}{ Secondary AML } & No & $23(61)$ & $78(61)$ & \multirow{2}{*}{0.964} \\
\hline & Yes & $15(39)$ & $50(39)$ & \\
\hline \multirow[t]{2}{*}{ Complete remission } & $C R$ & $28(74)$ & $110(86)$ & \multirow{2}{*}{0.077} \\
\hline & CRi & $10(26)$ & $18(14)$ & \\
\hline \multirow{2}{*}{$\begin{array}{l}\text { Conditioning } \\
\text { regimen }\end{array}$} & Myeloablative & $24(63)$ & $72(56)$ & \multirow{2}{*}{0.449} \\
\hline & Other & $14(37)$ & $56(44)$ & \\
\hline \multirow[t]{2}{*}{ Donor type } & Matched sibling & $12(32)$ & $42(33)$ & \multirow{2}{*}{0.887} \\
\hline & Other & $26(68)$ & $86(67)$ & \\
\hline \multirow{2}{*}{$\begin{array}{l}\text { Female donor: male } \\
\text { recipient (FDMR) }\end{array}$} & Other & $28(80)$ & $91(78)$ & \multirow{2}{*}{0.844} \\
\hline & FDMR & $7(2)$ & $25(22)$ & \\
\hline
\end{tabular}

$M R D$ minimal residual disease, $C R$ complete remission, $C R 1$ first $C R, A M L$ acute myeloid leukemia, $C R i C R$ with incomplete blood count recovery

were $M R D^{\text {neg }}$ had significantly longer RFS than patients who were $\operatorname{MRD}^{\text {pos }}(P=0.013)$. The median RFS was 1477 days for $\mathrm{MRD}^{\text {pos }}$ patients, and the median RFS in the $M D^{\text {neg }}$ group was not reached.

In univariate analysis, MRD detected by either MFC or CG/FISH was associated with inferior OS $(P=0.022$ and $P=0.0031$, respectively; Fig. 3 ) and the type of assay used to detect MRD did not appear to affect the outcome. Therefore, we grouped the MRD ${ }^{\text {pos }}$ patients together for survival outcome assessment.

We performed a multivariate analysis using a proportional sub-distribution hazards regression model and backward selection with RFS as the response variable (Table 3 ). The analysis revealed that only good or intermediate risk karyotype was associated with significantly better RFS (HR $=0.49$ (95\% CI: $0.27-0.87) ; P=0.016)$. 


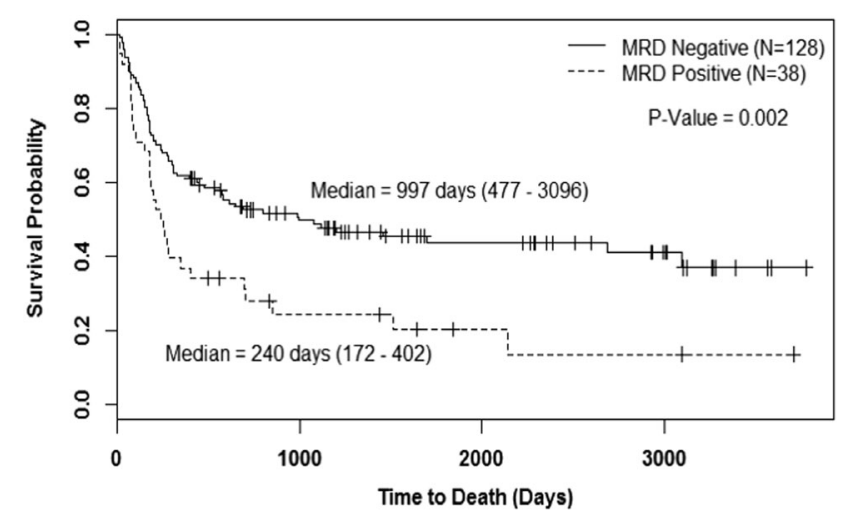

Fig. 1 Overall survival with any MRD. Overall survival based on any minimal residual disease (MRD) status irrespective of the type of MRD

Table 2 Multivariate analysis of factors affecting $O^{\mathbf{a}}$

\begin{tabular}{lllll}
\hline Variable & & HR & $\mathbf{9 5 \% ~ C l}$ & P-value \\
\hline MRD & Negative vs. positive & 0.553 & $0.351-0.871$ & 0.011 \\
Karyotype risk & $\begin{array}{l}\text { Good or intermediate } \\
\text { vs. poor risk }\end{array}$ & 0.512 & $0.331-0.791$ & 0.003 \\
$\begin{array}{l}\text { Complete } \\
\text { remission }\end{array}$ & Yes vs. no & 0.519 & $0.310-0.868$ & 0.013 \\
Secondary AML $^{\text {No vs. yes }}$ & 0.747 & $0.492-1.136$ & 0.173 \\
\hline
\end{tabular}

$A M L$ acute myeloid leukemia, $C I$ confidence interval, $H R$ hazard ratio, MRD minimal residual disease, OS overall survival

${ }^{\mathrm{a} B a c k w a r d}$ selection procedure was applied at the 0.2 significance level

${ }^{b}$ Complete remission vs. complete remission with incomplete count recovery

$\mathrm{MRD}^{\text {neg }}$ patients trended toward better RFS $(\mathrm{HR}=0.58$ (95\% CI: $0.32-1.08) ; P=0.087)$. In univariate analysis, MRD detected by either MFC or CG/FISH was associated with shorter RFS $(P=0.0059$ and $P=0.015$, respectively) as seen in Fig. 3.

\section{Non-relapse mortality}

There was no difference in the cumulative incidence of non-relapse mortality between $\mathrm{MRD}^{\text {pos }}$ and $\mathrm{MRD}^{\text {neg }}$ groups $(P=0.86)$ as shown in Fig. 4.

\section{Analysis of MRD pos patients}

Within the $\mathrm{MRD}^{\text {pos }}$ patient population, we compared those who survived more than 1 year to those who lived 1 year or less. Patient who lived longer than a year were less likely to have poor risk karyotype at baseline (29\% vs. $58 \%$ ), more often received a matched sibling donor graft ( $50 \%$ vs. $25 \%$ ), less often had MRD detected by persistent CG/FISH abnormalities (64\% vs. $75 \%$ ), less often received an ablative conditioning regimen ( $29 \%$ vs. $42 \%)$ and most importantly had a higher incidence of acute and chronic GVHD (86\% vs. 58\%). Based on these observations we analyzed GVHD in MRD ${ }^{\text {pos }}$ patients.

\section{Effect of GVHD among MRD ${ }^{\text {pos }}$ patients}

Results from the multivariate Cox proportional hazards model and backward selection procedure with RFS as the response variable are presented in Table 4a. The multivariate analysis revealed that the following variables were significantly associated with RFS: MRD by CG or FISH (HR $=0.051$ (95\% CI: 0.009-0.297); $P=0.0009)$, MRD by MFC (HR $=0.15$ (95\% CI: 0.04-0.569), $P=0.0053)$, the presence of acute GVHD (Grade $\geq 2$ ) or chronic GVHD $(\mathrm{HR}=0.053$ (95\% CI: 0.01-0.279), $P=0.0005)$.

The development of acute GVHD, chronic GVHD, either acute or chronic GVHD, and acute and chronic GVHD significantly improved OS $(P=0.02, P=0.0003, P$ $=0.03$, and $P=0.0001$, respectively). Results from the multivariate Cox proportional hazards model and backward selection procedure with OS as response variable are presented in Table 4b. CR status at HCT $(\mathrm{HR}=5.564$ (95\% CI: $1.671-18.527) ; P=0.0052), C R$ vs. CRi (HR = 0.12 (95\% CI: 0.035-0.414); $P=0.0008$ ), both acute (grade $\geq 2)$ and chronic GVHD (HR $=0.211$ (95\% CI: $0.081-0.547) ; P=0.0014)$, and donor status ( $\mathrm{HR}=0.257$, (95\% CI: $0.077-0.862) ; P=0.0277$ ) were significantly associated with OS.

\section{Discussion}

In this study, we demonstrated that the presence of MRD by MFC, CG, or FISH prior to HCT predicted inferior OS and RFS in AML patients. We also showed that the development of acute and chronic GVHD mitigated the adverse impact of detectable MRD on RFS and OS in these patients. Early studies have shown that evidence of MRD by flow cytometry after achieving a "morphological CR" predicts for an increased relapse rate and shorter RFS in AML patients ${ }^{21}$. Subsequent MRD studies post induction ${ }^{22,23}$ or post consolidation therapy confirmed poor RFS and OS in multivariate analysis irrespective of age $\mathrm{a}^{24,25}$. Poor AML outcomes were also 


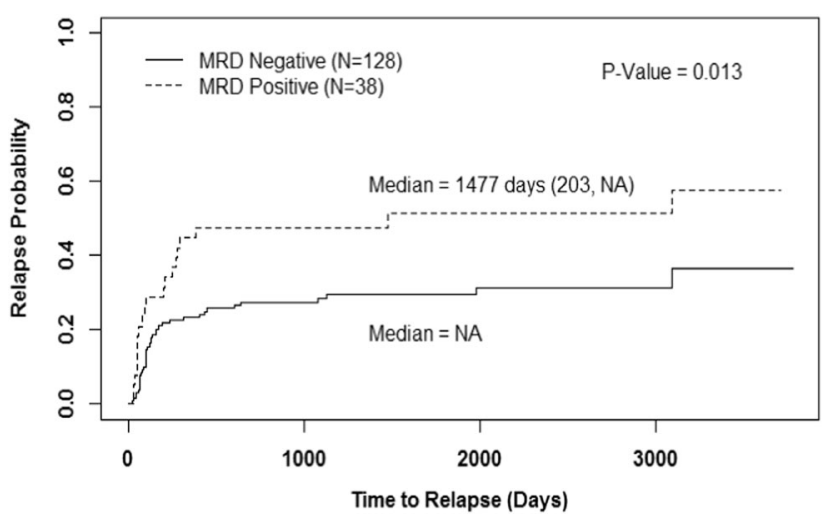

Fig. 2 Cumulative incidence of relapse with any MRD. Relapse-free survival based on any MRD status by method of cumulative incidence of relapse
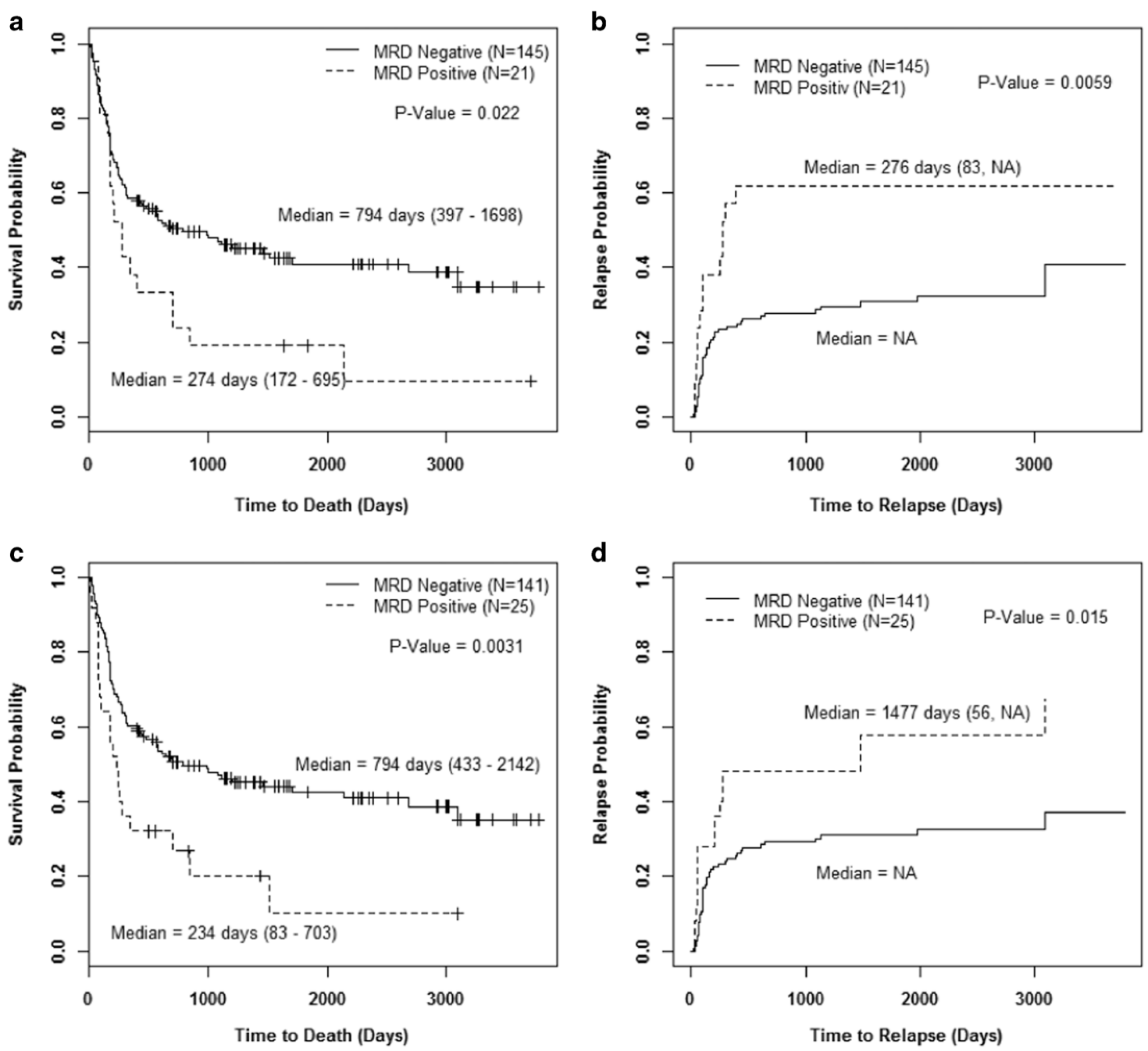

Fig. 3 Overall survival and cumulative incidence of relapse based on MRD by flow or cytogenetics/FISH. Overall survival and relapse-free survival by method of cumulative incidence of relapse assessed based on the type of minimal residual disease (MRD). a Overall survival based on MRD by flow. $\mathbf{b}$ Cumulative incidence of relapse based on MRD by flow. c Overall survival based on MRD by cytogenetics/FISH. $\mathbf{d}$ Cumulative incidence of relapse based on MRD by cytogenetics/FISH 
reported in patients with evidence of MRD by residual abnormal karyotype while in morphological remission ${ }^{23}$. Several recent retrospective single institution studies demonstrated the negative impact of $\mathrm{MRD}^{\text {pos }}$, assessed by 10-color MFC, prior to HCT on the probability of relapse in $A M L$ patients ${ }^{4-6}$. In one study, the $\mathrm{MRD}^{\mathrm{pos}}$ prior to HCT was as predictive of post-HCT relapse as the presence of active disease ${ }^{4}$. The significance of MRD assessed by CG or FISH on AML outcomes after HCT is not as clear. The published reports are limited in number and sample size, and have reported contradictory findings, with some reporting worse outcomes ${ }^{10,8}, 13$, and others reporting no significant difference ${ }^{5,11}$. Based on these

Table 3 Multivariate analysis of factors affecting $\mathbf{R F S}^{\mathbf{a}}$

\begin{tabular}{lllll}
\hline Variable & & HR & $\mathbf{9 5 \% ~ C l}$ & P-value \\
\hline MRD & Negative vs. positive & 0.584 & $0.316-1.081$ & 0.087 \\
Karyotype risk & $\begin{array}{l}\text { Better or } \\
\text { intermediate vs. poor } \\
\text { risk }\end{array}$ & 0.488 & $0.273-0.873$ & 0.016 \\
& $\begin{array}{l}\text { Matched sibling } \\
\text { donor vs. other }\end{array}$ & 0.639 & $0.356-1.146$ & 0.130 \\
Donor status & CR1 vs. >CR1 & 0.594 & $0.317-1.111$ & 0.100 \\
CR status at HCT & No vs. yes & 0.659 & $0.376-1.155$ & 0.140 \\
Secondary AML & Ablative vs. other & 0.627 & $0.361-1.088$ & 0.097 \\
Conditioning & & & & \\
regimen & & & &
\end{tabular}

$A M L$ acute myeloid leukemia, $C l$ confidence interval, $C R$ complete response, $H C T$ hematopoietic stem cell transplantation, $H R$ hazard ratio, MRD minimal residual disease, RFS relapse-free survival

${ }^{\mathrm{a} B a c k w a r d}$ selection procedure was applied at the 0.2 significance level reports, MRD by MFC appears to be clearly predictive of poor outcomes but the significance of karyotype needed further clarification.

In our study, we evaluated pre-HCT MRD by MFC and CG/FISH. When assessed separately on univariate analysis, MRD ${ }^{\text {pos }}$ status by either MFC or CG/FISH was associated with significantly worse RFS and OS. As outcomes did not differ by the method of MRD assessment and given the relatively small sample size, we combined all patients with any type of MRD positivity. Patients who were $M^{2} D^{\text {neg }}$ prior to HCT, by either MFC or CG/FISH, had significantly better OS and a trend toward better RFS on multivariate analysis. Our results suggest that evidence of MRD before HCT, regardless of the modality of testing used, predicts for poor AML outcomes.

It is well known that development of GVHD is associated with lower relapse rates in leukemia post $\mathrm{HCT}$, due to the GVL effect ${ }^{26}$. However, MRD ${ }^{\text {pos }}$ prior to HCT is still associated with very high relapse risk and inferior survival despite the development of GVHD. It is still unclear whether the GVL effect observed in patients with acute or chronic GVHD is capable of overcoming higher relapse rates observed in AML patients who are MRD ${ }^{\text {pos }}$ prior to HCT. Here we report a very important observation that the development of acute and chronic GVHD, and presumably its accompanying GVL effect, may overcome the adverse effect of MRD ${ }^{\text {pos }}$, at least in some patients.

The retrospective nature of our study has some limitations. As expected, more than half of our patients had a normal karyotype at diagnosis. MRD in these patients was therefore only evaluated by MFC, as we did not have access to PCR testing for MRD in many patients, who

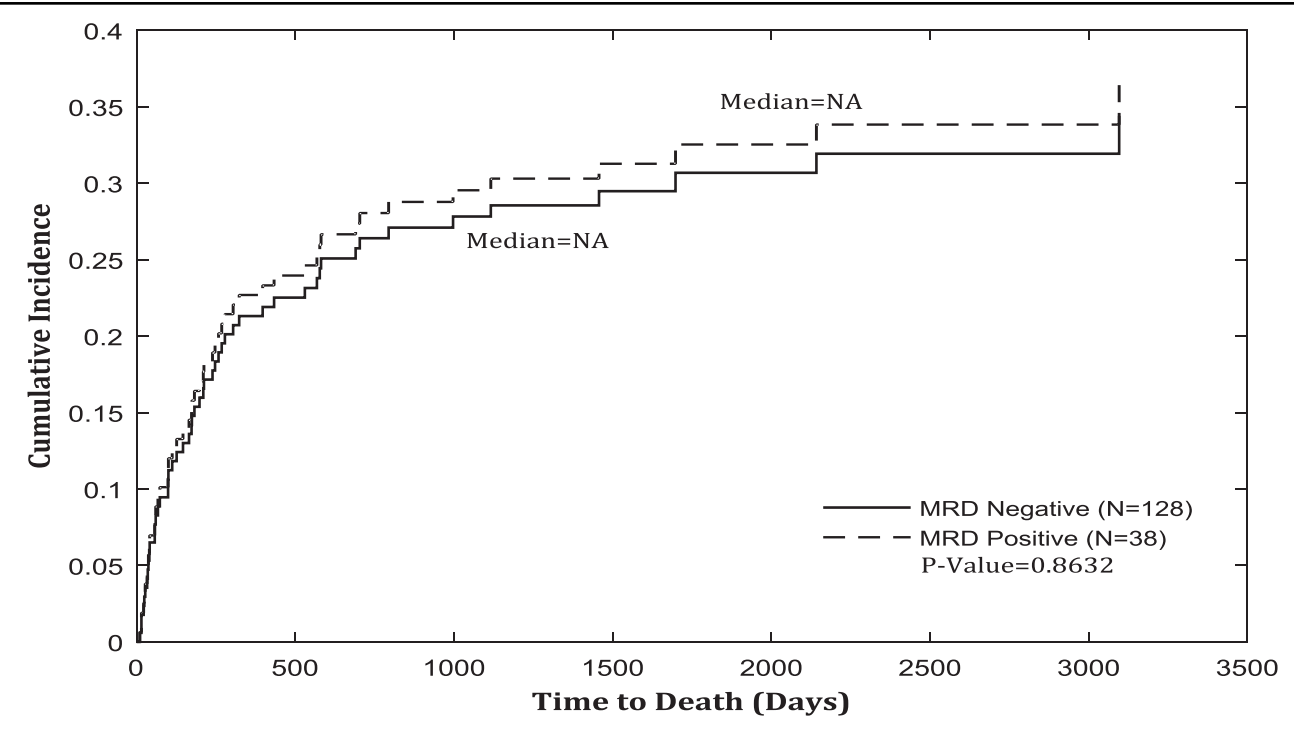

Fig. 4 Non-relapse mortality based on MRD status by method of cumulative incidence 
Table 4 Multivariate analysis of factors affecting $\operatorname{RFS}^{\mathrm{a}}$ (a) and $O S^{\mathrm{a}}$ (b) within MRD $^{\text {pos }}$ patients

\begin{tabular}{|c|c|c|c|c|}
\hline Variable & & HR & $95 \% \mathrm{Cl}$ & P-value \\
\hline Both acute (grade $\geq 2$ ) or chronic GVHD & Yes vs. no & 0.053 & $0.01-0.279$ & 0.0005 \\
\hline MRD by karyotype or FISH & Negative cytogenetics or FISH vs. positive cytogenetics or FISH & 0.051 & $0.009-0.297$ & 0.0009 \\
\hline MRD by flow cytometry & Negative vs. positive & 0.15 & $0.04-0.569$ & 0.0053 \\
\hline Remission status at $\mathrm{HCT}$ & CR vs. CRi & 5.824 & $0.639-53.107$ & 0.1182 \\
\hline Duration of $C R$ & $\geq 12$ months vs. $<12$ months & 0.333 & $0.068-1.633$ & 0.1753 \\
\hline Intensity of conditioning & Ablative vs. others & 2.497 & $0.665-9.375$ & 0.1753 \\
\hline
\end{tabular}

(b)

\begin{tabular}{lllll}
\hline Variable & & HR & 95\% Cl & P-value \\
\hline Type of CR & CR vs. CRi & 0.12 & $0.035-0.414$ & 0.0008 \\
Both acute (grade $\geq 2$ ) or chronic GVHD & Yes vs. No & 0.211 & $0.081-0.547$ & 0.0014 \\
CR status at HCT & CR1 vs. >CR1 & 5.564 & $1.671-18.527$ & 0.0052 \\
Donor type & MSD vs. Other & 0.257 & $0.077-0.862$ & 0.0277 \\
Karyotype Risk & Better or Intermediate vs. Poor Risk & 0.426 & $0.175-1.034$ & 0.0593 \\
MRD by flow cytometry & Negative vs. Positive & 0.493 & $0.173-1.405$ & 0.1855 \\
\hline
\end{tabular}

$C l$ confidence interval, $C R$ complete remission, $C R i C R$ with incomplete blood count recovery, FISH fluorescence in situ hybridization, $G V H D$ graft vs. host disease, $H R$ hazard ratio, MRD minimal residual disease, RFS relapse- free survival, HCT hematopoietic stem cell transplantation, MSD matched sibling donor

${ }^{a}$ Backward selection procedure was applied at the 0.2 significance level

were referred from outside hospitals. Although there are emerging data being accumulated about the prognostic significance of numerous molecular markers these tests are still not routinely utilized for MRD measurement in AML patients prior to HCT. In this study, we used only commonly accepted techniques for MRD monitoring such as MFC, CG, and FISH. The relatively small sample size did not allow us to analyze the statistical significance of residual disease detected separately by MFC or CG/ FISH in a multivariate analysis. Although small, our study has the second largest group of patients $(n=25)$ with residual CG/FISH. It will be useful to know the threshold of MRD (if one exists) above which the prognosis of AML is adversely affected. While earlier studies attempted to identify this threshold there is no consensus on this issue at this time ${ }^{24,} 25$. This could not be elucidated in our report as well.

Prior studies have reported improved outcomes in patients with MRD ${ }^{\text {pos 5, 7, 24, } 27}$ who proceed to HCT, but our study shows the prognosis of this subset of patients to be generally poor after HCT in comparison to patients with no MRD. This is in line with recent publications ${ }^{4-6}$. Importantly, we identified that development of acute or chronic GHVD can mitigate adverse effect of MRD ${ }^{\text {pos }}$ on survival. In can be explained that GVL effect associated with development of acute and chronic GVHD can successfully prevent disease relapse in some patents with $M R D^{\text {pos }}$. In agreement with prior reports, our study also showed that the intensity of the conditioning regimen did not have major effect on outcomes of $M R D^{\text {pos }}$ patients $^{5,28}$. Larger studies may help discern other differences among $M R D^{\text {pos }}$ patients with better survival vs. poorer survival and may also clarify if MRD has an additive role when present with other known poor prognostic risk factors.

\section{Conclusion}

MRD $^{\text {pos }}$ status prior to HCT, identified by either MFC, CG, or FISH, correlates with shortened survival times after HCT. MRD pos patients continue to have poor prognosis compared to $\mathrm{MRD}^{\text {neg }}$ patients despite HCT, although development of acute or chronic GVHD appears to have a positive impact on RFS and OS of MRD ${ }^{\text {pos }}$ patients compared to $\mathrm{MRD}^{\text {pos }}$ patients who do not develop acute or chronic GVHD.

\section{Author details}

${ }^{1}$ Division of Hematology and Oncology, Department of Internal Medicine, College of Medicine, University of Florida, Gainesville, FL, USA. ${ }^{2}$ Department of Biostatistics, College of Medicine, University of Florida, Gainesville, FL, USA. ${ }^{3}$ Department of Pathology, College of Medicine, University of Florida, Gainesville, FL, USA 


\section{Competing interests}

The authors declare that they have no competing interests.

\section{Publisher's note}

Springer Nature remains neutral with regard to jurisdictional claims in published maps and institutional affiliations.

\section{Supplementary information}

The online version of this article (https://doi.org/10.1038/s41408-017-0007-x) contains supplementary material.

\section{Received: 20 August 2017 Accepted: 25 August 2017}

Published online: 27 November 2017

\section{References}

1. Ferrara, F. \& Schiffer, C. A. Acute myeloid leukaemia in adults. Lancet $\mathbf{3 8 1}$ 484-495 (2013)

2. Kadia, T. M., Ravandi, F., Cortes, J. \& Kantarjian, H. Toward individualized therapy in acute myeloid leukemia: a contemporary review. JAMA Oncol. 1, 820-828 (2015).

3. Ravandi, F. Relapsed acute myeloid leukemia: why is there no standard of care? Best Pract. Res. Clin. Haematol. 26, 253-259 (2013).

4. Araki, D. et al. Allogeneic hematopoietic cell transplantation for acute myeloid leukemia: time to move toward a minimal residual disease-based definition of complete remission? J. Clin. Oncol. 34, 329-336 (2016).

5. Walter, R. B. et al. Comparison of minimal residual disease as outcome predictor for AML patients in first complete remission undergoing myeloablative or nonmyeloablative allogeneic hematopoietic cell transplantation. Leukemia 29, 137-144 (2015).

6. Zhou, Y. et al. Pre- and post-transplant quantification of measurable ('minimal') residual disease via multiparameter flow cytometry in adult acute myeloid leukemia. Leukemia 30, 1456-1464 (2016).

7. Chen, Y. et al. Persistence of cytogenetic abnormalities at complete remission after induction in patients with acute myeloid leukemia: prognostic significance and the potential role of allogeneic stem-cell transplantation. J. Clin. Oncol. 29, 2507-2513 (2011)

8. Walter, R. B. et al. Impact of pretransplantation minimal residual disease, as detected by multiparametric flow cytometry, on outcome of myeloablative hematopoietic cell transplantation for acute myeloid leukemia. J. Clin. Oncol. 29, 1190-1197 (2011).

9. Grubovikj, R. M., Alavi, A., Koppel, A., Territo, M. \& Schiller, G. J. Minimal residual disease as a predictive factor for relapse after allogeneic hematopoietic stem cell transplant in adult patients with acute myeloid leukemia in first and second complete remission. Cancers 4, 601-617 (2012).

10. Oran, B. et al. Significance of persistent cytogenetic abnormalities on myeloablative allogeneic stem cell transplantation in first complete remission. Biol. Blood Marrow Transplant. 19, 214-220 (2013).
11. Walter, R. B. et al. Significance of minimal residual disease before myeloablative allogeneic hematopoietic cell transplantation for AML in first and second complete remission. Blood 122, 1813-1821 (2013).

12. Ustun, C. et al. Achieving stringent $C R$ is essential before reduced-intensity conditioning allogeneic hematopoietic cell transplantation in AML. Bone Marrow Transplant. 48, 1415-1420 (2013).

13. Grimwade, D. et al. What happens subsequently in AML when cytogenetic abnormalities persist at bone marrow harvest? Results of the 10th UK MRC AML trial. Medical research council leukaemia working parties. Bone Marrow Transplant. 19, 1117-1123 (1997).

14. Cheson, B. D. et al. Revised recommendations of the International working group for diagnosis, standardization of response criteria, treatment outcomes, and reporting standards for therapeutic trials in acute myeloid leukemia. J. Clin. Oncol. 21, 4642-4649 (2003).

15. Hemmati, P. G. et al. A modified EBMT risk score predicts the outcome of patients with acute myeloid leukemia receiving allogeneic stem cell transplants. Eur. J. Haematol. 86, 305-316 (2011).

16. NCCN. Guidelines for Acute Myeloid Leukemia version 3.2017 (accessed October 2017).

17. Bacigalupo, A. et al. Defining the intensity of conditioning regimens: working definitions. Biol. Blood Marrow Transplant. 15, 1628-1633 (2009).

18. Gray, R. J. A class of K-sample tests for comparing the cumulative incidence of a competing risk. Ann. Stat. 16, 1141-1154 (1988).

19. Fine, J. P. \& Gray, R. J. A proportional haxzards model for subdistribution of a competing risk. J. Am. Stat. Assoc. 94, 496-509 (1999)

20. R Core Team. R: A Language and Environment for Statistical Computing ( Foundation for Statistical Computing, Vienna, Austria, 2015).

21. San Miguel, J. F. et al. Immunophenotyping investigation of minimal residual disease is a useful approach for predicting relapse in acute myeloid leukemia patients. Blood 90, 2465-2470 (1997).

22. Freeman, S. D. et al. Prognostic relevance of treatment response measured by flow cytometric residual disease detection in older patients with acute myeloid leukemia. J. Clin. Oncol. 31, 4123-4131 (2013).

23. Marcucci, G. et al. Abnormal cytogenetics at date of morphologic complete remission predicts short overall and disease-free survival, and higher relapse rate in adult acute myeloid leukemia: results from cancer and leukemia group B study 8461. J. Clin. Oncol. 22, 2410-2418 (2004).

24. Maurillo, L. et al. Toward optimization of postremission therapy for residual disease-positive patients with acute myeloid leukemia. J. Clin. Oncol. 26, 4944-4951 (2008).

25. Terwijn, M. et al. High prognostic impact of flow cytometric minimal residual disease detection in acute myeloid leukemia: data from the HOVON/SAKK AML 42A study. J. Clin. Oncol. 31, 3889-3897 (2013).

26. Barrett, A. J. \& Battiwalla, M. Relapse after allogeneic stem cell transplantation Expert Rev. Hematol. 3, 429-441 (2010).

27. Laane, E. et al. The effect of allogeneic stem cell transplantation on outcome in younger acute myeloid leukemia patients with minimal residual disease detected by flow cytometry at the end of post-remission chemotherapy. Haematologica 91, 833-836 (2006).

28. Anthias, C. et al. Pre-transplant MRD predicts outcome following reducedintensity and myeloablative allogeneic hemopoietic SCT in AML. Bone Marrow Transplant. 49, 679-683 (2014). 